\title{
Position Drift Compensation in Port-Hamiltonian Based Telemanipulation
}

\author{
Cristian Secchi ${ }^{1}$, Stefano Stramigioli ${ }^{2}$, Cesare Fantuzzi ${ }^{1}$ \\ ${ }^{1}$ DISMI, Univ. of Modena and Reggio Emilia \\ Viale Allegri 13, 42100 Reggio Emilia, Italy \\ \{secchi.cristian,fantuzzi.cesare\}@unimore.it \\ ${ }^{2}$ EEMCS, University of Twente \\ P.O.Box 217, 7500 AE Enschede, NL \\ S.Stramigioli@ieee.org
}

\begin{abstract}
Passivity based bilateral telemanipulation schemes are often subject to a position drift between master and slave if the communication channel is implemented using scattering variables. The magnitude of this position mismatch can be significant during interaction tasks. In this paper we propose a passivity preserving scheme for compensating the position drift arising during contact tasks in port-Hamiltonian based telemanipulation improving the kinematic perception of the remote environment felt by the human operator.
\end{abstract}

\section{INTRODUCTION}

Passivity theory is a very suitable tool for the implementation of bilateral telemanipulation schemes over delayed communication channels. In [1], a generic framework for geometric telemanipulation of port-Hamiltonian systems ([2]) has been proposed; master and slave are controlled through intrinsically passive port-Hamiltonian regulators which allow to shape the energetic behavior of the robots and to achieve desired dynamic properties at master and slave sides. Local and remote sides are interconnected through a scattering based communication channel that allows a lossless exchange of energy independently of any constant delay. In [3] discrete scattering is used to build a control scheme for telemanipulation of port-Hamiltonian systems over a packet switched communication network (e.g. Internet). The overall telemanipulation system is passive and, therefore, characterized by a stable behavior. When using scattering based communication channels, local and remote sides exchange only velocity and force information and this can cause the rise of a position drift between master and slave robots as reported in [4], [5], [6]. In particular, during interaction tasks, the communication delay induces a delay in the equilibration of the force applied by the human and, consequently, at steady state, a mismatch between the master and slave positions. Thus, the user feels the remote environment as if it was at a different position and this negatively affects the kinematic feedback perceived. Several works addressed the problem of position tracking in telemanipulation. In [5] an outer position loop is added to decrease the position error between master and slave; in [6] a novel control scheme for position regulation achieved passifying the control and the communication block is proposed.

A very effective approach for position drift compensation is that proposed in [7] where a linear telemanipulator with a linear intrinsically passive controllers (IPC, [8]) is considered. The controller can be modeled as a virtual physical system that is endowed with a virtual spring with a variable rest length; changing the rest length of the spring allows compensating the position offset between master and slave which is explicitly computed solving the equations describing the telemanipulation system. The introduction of a variable rest length element does not change the kind of dynamic behavior of the controller but it simply introduces an offset; consequently, the user keeps on perceiving the same kind of impedance and he/she can keep on using the telemanipulation system in the same way but with the advantage of a correct kinematic feedback of the remote environment. In order to preserve passivity, part of the energy introduced into the system by the human operator is used for the compensation process. This represents a drawback for the proposed scheme since during the compensation phase the user perceives a spurious dynamic effect (due to the energy deviation) which can distract him/her deteriorating the usability of the telemanipulator. Furthermore, as shown in [7], the fact that the compensation process depends on the amount of energy injected by the user into the system can lead to slow transients in the rest length variation which yield poor compensation performances.

In this paper we address the problem of compensating the position offset that takes place during contact tasks in portHamiltonian telemanipulation over delayed communication channels. Following and generalizing the idea proposed in [7], we endow one of the elastic elements that characterize the port-Hamiltonian controller with a variable rest length which allows to compensate the position drift introduced by the scattering based communication channel. In order to implement the compensation in a passive way without directly requiring the human intervention, we modify the port-Hamiltonian controller at the slave side replacing the dissipative element needed to implement impedance matching with a storing element, the tank, that allows to store the energy that has to be absorbed to match the impedance of the communication channel. The content of the tank is then used for changing the rest length and for compensating the position drift. In this way, the compensation is performed internally to the controller without requiring the human to keep on injecting energy into the system. Furthermore, since the controller can 
freely exploit the energy stored into the tank, there are no limitations on the transient behavior that can be imposed to the rest length variation. Finally, the proposed control strategy is valid for general port-Hamiltonian controllers and it can be applied also to nonlinear telemanipulation systems. Once the offset is compensated the user perceives the remote environment at its real, non biased, position despite of the presence of the delayed communication channel. In this way the telemanipulation system provides to the user both a force feedback AND a kinematic feedback of the environment improving, therefore, the realism of the remote interaction experienced by the operator at the local side.

The paper is organized as follows. In Sec. II some background is given on port-Hamiltonian based telemanipulation and in Sec. III we will illustrate how to modify the slave controller for storing the energy that will be exploited for the position drift compensation. In Sec. IV the overall control scheme is illustrated and in Sec. V some simulations are provided to validate the results of the paper. Finally in Sec. VI some conclusions are drawn and future work is addressed.

\section{BACKGROUND}

We can consider a port-Hamiltonian system as composed of a state manifold $\mathcal{X}$, a lower bounded energy function $H: \mathcal{X} \rightarrow \mathbb{R}$ corresponding to the internal energy, a network structure, represented by a skew-symmetric matrix, $D(x)=$ $-D^{T}(x)$ whose graph has the mathematical structure of a Dirac structure, which is in general a state dependent power continuous interconnection structure, and an interconnection port represented by a pair of dual power variables $(e, f) \in$ $V^{*} \times V$ called effort and flow respectively. This port is used to interact energetically with the system: the power supplied through a port is equal to $e^{T} f$. We can furthermore split the interaction port in more sub-ports, each of which can be used to model different power flows. We will indicate with the subscript $I$ the power ports by means of which the system interacts with the rest of the world, with the subscript $C$ the power ports associated with the storage of energy and with the subscript $R$ the power ports relative to power dissipation. Summarizing, we have:

$$
\left(\begin{array}{c}
e_{I} \\
f_{C} \\
e_{R}
\end{array}\right)=D(x)\left(\begin{array}{c}
f_{I} \\
e_{C} \\
f_{R}
\end{array}\right)
$$

where $D(x)$ is a skew symmetric matrix representing the Dirac structure. Loosely speaking, a port-Hamiltonian system is made up of a set of energy processing elements (energy storing, energy dissipating and sources of energy) that exchange energy by means of their power ports through a set of energy paths which form a power preserving interconnection that can be modeled as a Dirac structure.

The dissipation in the system can be modeled using as characteristic equations $e_{R}=R(x) f_{R}$ with $R(x)$ a symmetric and positive semi-definite matrix; in this way $e_{R}^{T} f_{R} \geq 0$ meaning that power is always absorbed by the dissipating elements. If we furthermore set $\dot{x}=f_{C}$ and $e_{C}=\frac{\partial H}{\partial x}$,

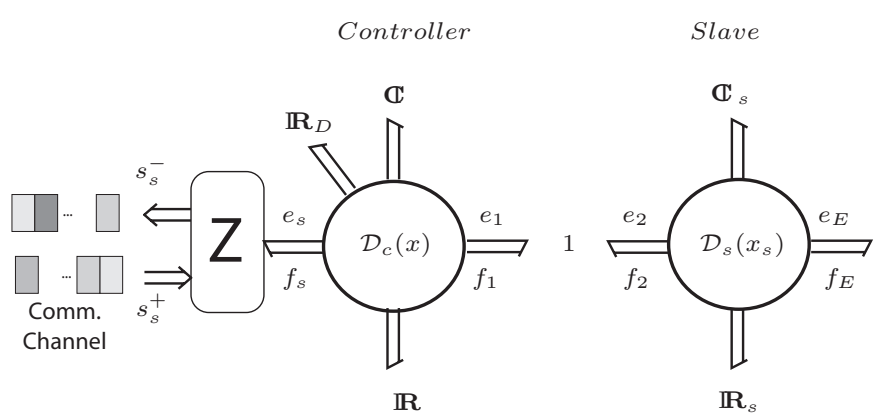

Fig. 1. The Port-Hamiltonian based Telemanipulation Scheme.

due to the skew-symmetry of $D(x)$, we can easily see that $\dot{H}+f_{R}^{T} R(x) f_{R}=e_{I}^{T} f_{I}$, which clearly states that the supplied power $e_{I}^{T} f_{I}$ equals the increase of internal energy plus the dissipated one and that, therefore, a port-Hamiltonian system is passive.

A very broad class of physical systems, both linear and non linear, can be modeled within the port-Hamiltonian framework which can therefore be used to model telemanipulation systems endowed with nonlinear robots. For further information the reader is addressed to [2].

In port-Hamiltonian based telemanipulation, both master and slave robots can be modeled as port-Hamiltonian systems. The slave is interconnected in a power preserving way to a port-Hamiltonian controller which acts as an intrinsically passive impedance controller [8]. Master and slave sides exchange power through a transmission line that is characterized, in general, by a non negligible transmission delay. Each power port by means of which master and slave sides exchange power through the communication channel is characterized by an effort $e(t)$ and by a flow $f(t)$ and it can be equivalently represented by an incoming power wave $s^{+}(t)$ and an outgoing power wave $s^{-}(t)$ defined as

$$
\left\{\begin{array}{l}
s^{+}(t)=\frac{1}{\sqrt{2}} N^{-1}(e(t)+Z f(t)) \\
s^{-}(t)=\frac{1}{\sqrt{2}} N^{-1}(e(t)-Z f(t))
\end{array}\right.
$$

where $Z=N N>0$ is the symmetric positive definite impedance of the scattering transformation. In order to get a passive exchange of energy independently of any constant communication delay, the power ports connected to the transmission line are decomposed into a pair of scattering variables which are transmitted along the channel. In the considered scheme, we use only one impedance controller at the slave side, as recently proposed in [4], instead of two impedance controllers, as proposed in [1], [3]. The controller is used to impose the impedance perceived by the user and it can be physically interpreted as a set of (possibly non linear, [9]) elastic, inertial and dissipative elements interconnected together. The slave side of the port-Hamiltonian based bilateral telemanipulation scheme is represented in Fig. 1 in a bond-graph notation. In order to avoid the wave reflection phenomenon [10], [1], which arises when using scattering based communication channels and which highly degrades 
performances, the controller must be endowed with a dissipative element $\left(\mathbb{R}_{D}\right)$ which implements the so called impedance matching. This element must be characterized by the following port behavior:

$$
e_{D}=Z f_{D}
$$

where $\left(e_{D}, f_{D}\right)$ is the power port that characterizes the dissipative element, $f_{D}=f_{s}$ and $Z$ is the impedance of the scattering transformation.

Since a port-Hamiltonian based bilateral telemanipulator is made up of passive subsystems interconnected in a power preserving way, the overall system is intrinsically passive and, therefore, characterized by a stable behavior. For further details the reader is addressed to [1], [3].

\section{IMPLEMENTATION OF THE ENERGY TANK}

The physical role of the dissipating element added to the controller to implement impedance matching, is to absorb the energy content of the scattering wave that otherwise would be reflected back to the master side. The goal of this section is to replace this dissipative element with a storing element, that we call tank, which keeps on absorbing energy implementing the behavior reported in Eq.(3) (and that, therefore, keeps on implementing the impedance matching) but that, additionally, stores the absorbed energy that can be used for other control purposes (e.g. for compensating the position error between master and slave, as explained in the next section).

The port behavior of an energy storing element is described by

$$
\left\{\begin{array}{l}
\dot{x}_{T}=f_{C} \\
e_{C}=\frac{\partial H_{T}}{\partial x_{T}}
\end{array}\right.
$$

where $\left(e_{C}, f_{C}\right)$ is the power port through which the element exchanges energy and $H_{T}(\cdot)$ is a lower bounded function corresponding to the stored energy. If we simply replaced the dissipative element $\mathbb{R}_{D}$ with a storing element we wouldn't achieve the port behavior described by Eq.(3) and, consequently, we wouldn't match the impedance of the communication channel. This is mainly due to the fact that energy storing elements and energy dissipating elements process energy in two different ways: the first perform a reversible energy transformation (i.e. energy can both be absorbed and released) while the latter perform an irreversible energy transformation (i.e. energy can travel only in one direction).

The idea for implementing both energy storage and impedance matching is to modify the interconnection structure that joins the tank to the rest of the controller to force energy absorption and to impose the port behavior reported in Eq.(3).

In the following we will indicate with $\operatorname{diag}\left(a_{i}\right), i=$ $1, \cdots, n$ the $n \times n$ diagonal matrix whose diagonal is made up by the elements $a_{1} \ldots a_{n}$. Furthermore, we will drop the dependence on time of efforts and flows to lighten the notation.

Proposition 1: Consider the control structure reported in Fig. 2 where $\mathbb{M} \mathbb{M} \mathbb{F}$ represents the following modulation law:

$$
\left\{\begin{array}{l}
f_{T}=m_{b} f_{D} \\
e_{D}=m_{b}^{T} e_{T}
\end{array} \quad m_{b}=\operatorname{Zdiag}\left(\frac{f_{D_{i}}}{e_{T_{i}}}\right) \quad i=1, \ldots, n\right.
$$

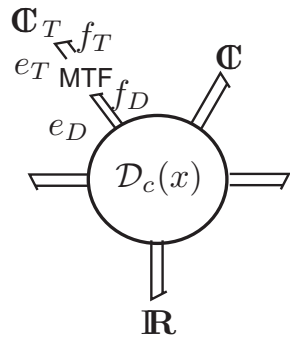

Fig. 2. The modified controller

where $f_{D_{i}}$ and $e_{T_{i}}$ represent the $i^{\text {th }}$ component of the vectors $f_{D}$ and $e_{T}$ respectively. If $e_{T_{i}} \neq 0$, the tank element absorbs energy and the behavior implemented at the port $\left(e_{D}, f_{D}\right)$ is that reported in Eq.(3).

Proof: We first prove that energy is always absorbed by the tank. The power flowing into the tank is given by $e_{T}^{T} f_{T}$. Using Eq.(5) we have that:

$$
e_{T}^{T} f_{T}=e_{T}^{T} m_{b} f_{D}=e_{T}^{T} Z \operatorname{diag}\left(\frac{f_{D_{i}}}{e_{T_{i}}}\right) f_{D}
$$

since $Z$ is symmetric and positive definite we can write:

$$
e_{T}^{T} f_{T}=\underbrace{e_{T}^{T} \operatorname{diag}\left(\frac{f_{D_{i}}}{e_{T_{i}}}\right)}_{f_{D}^{T}} Z f_{D}=f_{D}^{T} Z f_{D}>0
$$

Thus, power is always flowing into the tank and, therefore, the tank always absorbs energy. Furthermore we have that

$$
e_{D}=m_{b}^{T} e_{T}=\operatorname{diag}\left(\frac{f_{D_{i}}}{e_{T_{i}}}\right) Z^{T} e_{T}=Z \underbrace{\operatorname{diag}\left(\frac{f_{D_{i}}}{e_{T_{i}}}\right) e_{T}}_{f_{D}}=Z f_{D}
$$

which proves that the behavior implemented at the port $\left(e_{D}, f_{D}\right)$ is that reported in Eq.(3)

The role of the interconnection used to connect the tank to the rest of the controller is to transform a reversible element into an irreversible one. Loosely speaking, the role of the modulation is to mask the port behavior of the tank element to make it appear as a dissipative element which matches the impedance of the communication channel. At the same time, since the tank is a storing element, the energy absorbed is not dissipated but stored.

Remark 1: In case $e_{T_{i}}=\frac{\partial H_{T}}{\partial x_{T_{i}}}=0$, the modulation reported in Eq.(5) is not well defined. This situation can be avoided by choosing, for example, an energy tank characterized by a function $1 / 2 x_{T}^{T} K x_{T}$ with $K>0$ and by precharging the tank by considering an initial configuration $x_{T}$ such that $x_{T_{i}}>0 \forall i$. In this way, since the tank always absorbs energy following Eq.(3), it is impossible that one of the components of $e_{T}$ goes to zero.

Since their port behavior is the same, the user perceives the dissipative element used to implement the impedance matching proposed in [1] and the energy tank in the same way. Thus the energy storing process is completely hidden to the user that can keep on using the system without taking care of the 
modification made on the control structure and without feeling any spurious dynamic effect while charging the tank.

Finally, let us remark that the modulation reported in Eq.(5) is power preserving since it can be easily seen that $e_{T}^{T} f_{T}=$ $e_{D}^{T} f_{D}$ and therefore we can embed it in the interconnection structure that connects all the energy processing elements of the controller achieving a new augmented Dirac structure $\mathcal{D}_{c a}\left(x, x_{T}\right)$. Thus the modified controller can still be modeled as a passive port-Hamiltonian system.

\section{PASSIVE COMPENSATION OF THE POSITION DRIFT}

In port-Hamiltonian telemanipulation, the controller always contains at least one elastic element which is used to implement the stiffness of the impedance perceived by the human operator [1], [3]. If we want to consider variable rest length elements, we can model the rest length $l$ as a state variable and endow the element with an extra power port $\left(e_{L}, f_{L}\right)$ through which it is possible to exchange energy for modifying $l$ [9]. Thus the behavior of a variable rest length spring is represented by

$$
\left\{\begin{array}{l}
\dot{x}(t)=f(t) \\
\dot{l}(t)=f_{L}(t) \\
e(t)=\frac{\partial H}{\partial x} \\
e_{L}(t)=\frac{\partial H}{\partial l}
\end{array}\right.
$$

where $(e, f)$ is the power port associated to the deformation of the spring, represented by the state variable $x$.

In [4] it is shown that, during contact tasks, the communication channel characterized by a transmission delay $T$ can be modeled as a spring with stiffness $K_{c h}=\frac{1}{T} Z$, where $Z$ is the impedance of the scattering transformation.

The force information is fed back to the master side through the transmission line and thus, at steady state, the position drift that takes place between master and slave sidesis given by:

$$
\Delta=T Z^{-1} e_{e n v}
$$

where $e_{e n v}$ is the effort applied by the remote environment. Thus, during contact tasks, the user perceives the remote environment as if it was in a different position. To give a proper perception, at steady state, for the same force on both sides the positions of master and slave should be the same. The idea for eliminating this drift is to introduce an offset that compensates it by changing the rest length of the elastic element of the controller. In this way, loosely speaking, the controller "pushes" the master robot to a configuration equal to that of the slave improving the kinematic perception of the remote environment felt by the user. Once the compensation has been done, the user can interact with the environment (e.g. sliding over a surface while applying a certain force) that is felt at its effective position at the master side.

To change the rest length, it is necessary to exchange energy via the length port $\left(e_{L}, f_{L}\right)$. In order to preserve the passivity of the overall scheme we do not have to inject extra energy into the system for the compensation process and, therefore, we want to use the energy stored into the tank we described in Sec. III. Thus, it is necessary to be able to drive energy from the tank to the length port and viceversa. The following result is useful:

Proposition 2: Let $\left(e_{T}, f_{T}\right)$ and $\left(e_{L}, f_{L}\right)$ be the tank port and the length port respectively and interconnect them with the Dirac structure $\mathcal{D}_{T}$ described by

$$
\left(\begin{array}{l}
f_{T} \\
f_{L}
\end{array}\right)=\left(\begin{array}{cc}
0 & -m_{t} \\
m_{t} & 0
\end{array}\right)\left(\begin{array}{l}
e_{T} \\
e_{L}
\end{array}\right)
$$

where $m_{t}=\operatorname{diag}\left(\gamma_{i} e_{L_{i}} e_{T_{i}}\right) \quad i=1 \ldots n$.

If $\gamma_{i}>0 \forall i$ then energy is extracted from the tank and injected into the length port while if $\gamma_{i}<0 \forall i$ then energy is extracted from the length port and injected into the tank.

Proof: Using Eq.(11), we have that

$$
e_{L}^{T} f_{L}=e_{L}^{T} \operatorname{diag}\left(\gamma_{i} e_{L_{i}} e_{T_{i}}\right) e_{T}=\sum_{i=0}^{n} \gamma_{i}\left(e_{L_{i}}\right)^{2}\left(e_{T_{i}}\right)^{2}
$$

and that

$$
e_{L}^{T} f_{L}=e_{L}^{T} m_{t} e_{T}=-e_{T}^{T} f_{T}
$$

Thus, from Eq.(12) and Eq.(13), we have that if $\gamma_{i}>0 \forall i$, then $-e_{T}^{T} f_{T}=e_{L}^{T} f_{L}>0$ which means that energy is extracted from the tank port and injected in the length port. Instead, if $\gamma_{i}<0 \forall i$ we have that $-e_{T}^{T} f_{T}=e_{L}^{T} f_{L}<0$ which means that energy is extracted from the length port and injected into the tank port.

Thus, introducing the Dirac structure described in Eq.(11) between the tank port and the length port it is possible to drive the energy flow by properly setting the control parameter $\gamma$. The signs of the elements of $\gamma$ determine the direction of the power transfer while their magnitudes can be used to boost the power transfer allowing us to achieve an energy transfer which is as fast as desired.

In order to eliminate the position drift introduced by the scattering based communication channel, we need to introduce an offset that compensates what is reported in Eq.(10). Thus, the target rest length of the elastic element introduced in the controller has to be set to

$$
l_{T}=-T Z^{-1} e_{e n v}
$$

where $T$ is the communication delay, $Z$ is the impedance of the scattering transformation and $e_{e n v}$ is the effort exchanged with the environment that can be measured by means of force sensors on the slave robot.

For each component of the rest length, we need to properly set the control parameters $\gamma_{i}$ depending on whether energy has to be extracted from or supplied to the length port to reach the target.

Using Eq.(9) it is possible to see that if $l_{i}<l_{T_{i}}$, then the $i^{\text {th }}$ component of the length has to increase and therefore, it is necessary to set $f_{L_{i}}>0$. If $\frac{\partial H}{\partial l_{i}}>0$, requiring $f_{L_{i}}>0$ means requiring a positive power flow towards the length port and thus, in this case, it is necessary to set $\gamma_{i}>0$. On the other hand, if $\frac{\partial H}{\partial l_{i}}<0$, requiring $f_{L_{i}}>0$ means requiring a negative power flow towards the length port or, equivalently, a positive power flow towards the tank; thus in this case it is necessary to set $\gamma_{i}<0$. Similar considerations hold in case it 


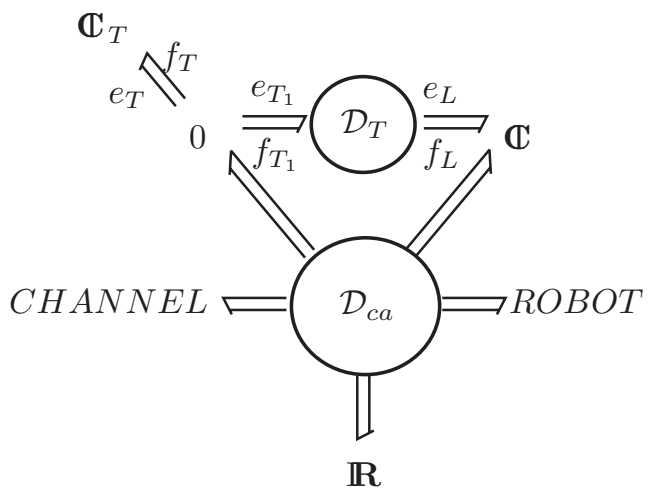

Fig. 3. The global port-Hamiltonian controller

is required $f_{L_{i}}<0$. Thus, once the sign of the components of $f_{L}$ is determined by comparing $l$ with $l_{T}$, it is necessary to check the sign of the components of $\frac{\partial H}{\partial l}$ to determine the sign of the transfer parameter $\gamma$. Summarizing, the following tuning algorithm can be used:

- If $\operatorname{sign}\left(l_{T_{i}}-l_{i}\right)=\operatorname{sign}\left(\frac{\partial H}{\partial l_{i}}\right)$ then $\gamma_{i}>0$

- If $\operatorname{sign}\left(l_{T_{i}}-l_{i}\right) \neq \operatorname{sign}\left(\frac{\partial H}{\partial l_{i}}\right)$ then $\gamma_{i}<0$

Remark 2: The proposed tuning strategy can give rise to chattering phenomena which can degrade the perception felt by the user. This can be avoided using standard control techniques as boundary layers [11].

We can now put together the tank implementation described in Sec. III and the energy transfer mechanism described in Eq.(11) to get the overall controller which is represented in Fig. 3 in a bond graph notation. We can distinguish two Dirac structures: $\mathcal{D}_{c a}$ and $\mathcal{D}_{T}$ interconnected in a power preserving way. The first derives directly from the port-Hamiltonian impedance control which implements the dynamics perceived by the user augmented by the modulation reported in Eq.(5). The embedding of the modulation is not perceived by the user and it allows to store the energy that would otherwise be dissipated during the impedance matching process. The interconnection structure $\mathcal{D}_{T}$ is what we call the transfer Dirac structure, namely the modulated interconnection structure described by Eq.(11) that allows us to control the energy transfer between the tank and the length port of the elastic element.

Remark 3: When extracting energy from the tank, it is necessary to be careful. In fact, it is necessary to leave some energy into the tank to avoid the problems reported in Remark 1. This can be done by setting an energy threshold below which energy extraction is forbidden.

Intuitively, the overall controller is passive since the energy used for implementing the position drift compensation is that stored into the energy tank which comes from the impedance matching process. More formally, intrinsic passivity of the controller can be proven by recalling that a power preserving interconnection of two Dirac structures is still a Dirac structure
[2]. Thus, the power preserving interconnection of $\mathcal{D}_{c a}$ and $\mathcal{D}_{T}$ gives a Dirac structure and, therefore, the overall controller is again a power preserving interconnection of energy storing elements, energy dissipating elements and interaction ports. Therefore the controller is still an intrinsically passive portHamiltonian system. Notice that the design of the impedance control and the embedding of the intrinsically passive position drift compensation can be implemented independently. In fact, the position drift compensation structure, is built upon the impedance controller using the energy that would be dissipated during the impedance matching process. Thus, given a generic port-Hamiltonian impedance control for telemanipulation, we can always improve the control performances by embedding the position drift compensation mechanism without altering the kind of dynamic behavior that is perceived by the user. One last question remains to be answered: is the energy stored in the tank always enough to change the rest length of the desired amount? Reasonably, we can assume that the force that the human operator can apply to the master is bounded. Thus, since the overall system is passive, the effort $e_{e n v}$ that the environment exchange with the slave robot is limited and, consequently, the maximum amount of energy $E_{0}>0$ required for performing the position drift compensation is also limited. If the energy stored in the tank is greater than $E_{0}$ we can always perform the compensation. In fact, in the worst case, when the user is applying the maximum effort to the master, all the energy required for the compensation can be extracted by the tank. When the user decreases the force applied, the position drift diminishes and some energy is released back into the tank. Finally, when the slave is no more in contact with the environment, all the energy that has been used for the compensation is released back to the tank which has again, enough energy for performing any another position drift compensation. Loosely speaking, since the energy transfer that takes place through the transfer Dirac structure is reversible and controlled, it is possible to recover the energy used for compensation when it isn't necessary anymore. Thus, if an energy amount sufficient for the worst case compensation is available, we are ensured that we can eliminate the position offset introduced by the communication channel in any case. If the energy stored in the tank is less than $E_{0}$, it can happen that only a partial compensation can be achieved and that performances are only partially improved. Nevertheless, while the human operator is using the system, the tank is continuously filled and, at the end, it will be storing an energy amount greater that $E_{0}$ and all the required compensations will be fully achievable.

\section{Simulations}

The aim of this section is to provide some simulations in order to validate the results of the paper. We consider a simple one degree of freedom telemanipulator where master and slave are simple masses of $0.5 \mathrm{Kg}$. The slave is interconnected to a port-Hamiltonian impedance controller and local and remote sides are joined through a scattering based communication channel characterized by an impedance $Z=1$ and by a 

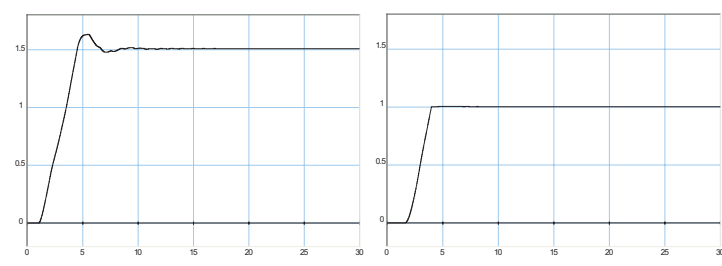

(a) Master position with PD (b) Slave position with $\mathrm{PD}$ controller controller
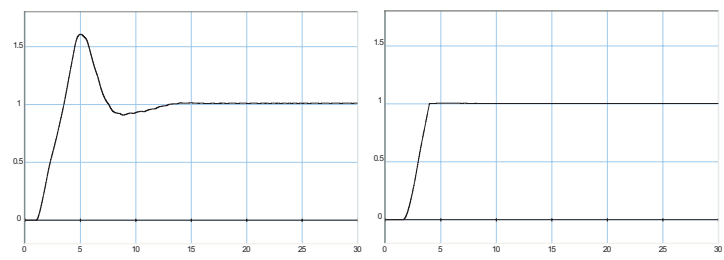

(c) Master position with the (d) Slave position with the proproposed controller posed controller

Fig. 4. Comparison of master and slave positions in case PD controller and the proposed control strategy are used during a contact task

transmission delay $T=0.5 \mathrm{~s}$ in both senses of communication. The port-Hamiltonian controller is a simple PD, physically equivalent to the parallel of a spring with stiffness $K=200 \mathrm{~N} / \mathrm{m}$ and a damper with dissipation coefficient $b=$ $1 \mathrm{~N} \mathrm{sec} / \mathrm{m}$ used to match the impedance of the communication channel. We consider a contact task and we compare the behaviors of the system when using a PD controller and the proposed control scheme. The user applies a constant force $e_{H}=1 \mathrm{~N}$ and the slave gets in contact with a rigid viscoelastic wall characterized by a stiffness $K_{w}=5000 \mathrm{~N} / \mathrm{m}$ and by a damping $b_{w}=40 \mathrm{Ns} / \mathrm{m}$ at position $x=1 \mathrm{~m}$. Master and slave positions are reported in Fig. 4. The slave robot stops when it meets the wall and the interaction force is fed back to the master side. In case the PD controller is adopted (Fig. 4(a) and Fig. 4(b)) we can see that the interaction force is fed back to the master side through the transmission line and that it equilibrates the force applied by the user. Nevertheless, because of the scattering based nature of the communication channel, a significant position drift exists between master and slave position at the equilibrium. Thus, the user experiences a good force feedback but a bad kinematic feedback. If the proposed control scheme is used, the interaction force is still fed back to the master side and it equilibrates the force applied by the user implementing the same force feedback as in the case PD control is used. Furthermore, when the slave gets in touch with the environment, the rest length of the spring is changed to introduce an offset that compensates that introduced by the communication channel; the compensation is passivity preserving since it uses the energy that is stored in the tank through the impedance matching process. We can see in Fig. 4(c) and Fig. 4(d) that the position drift between master and slave drastically decreases giving thus to the user both a good force feedback and a good kinematic feedback. Loosely speaking, the variation of the rest length pushes back the physical system made up by the master robot and by the communication channel and, therefore, the force feedback perceived by the user is given by the sum of two contribution: the first encodes the information relative to the interaction with the environment and the second derives from the compensation process. When the latter contribution vanishes the force feedback is exactly the same as that which would be achieved using a PD controller but the position drift between master and slave is eliminated.

\section{CONCLUSIONS AND Future WORK}

In this paper we have proposed a novel control algorithm for port-Hamiltonian based bilateral telemanipulation. It allows compensating the position drift introduced by the scattering based communication channel during contact tasks while preserving passivity of the overall scheme without requiring the direct intervention of the user. The proposed control structure is an extension of the existing port-Hamiltonian controller and its action on the system is minimal. The user can't distinguish the port-Hamiltonian controller from the augmented controller in case of free motion. The effect of the new control structure is active only during the contact phase, when the compensating offset is introduced. Furthermore, properly tuning of the energy transfer between the tank and the length port, it is possible to achieve any desired transient behavior in the rest length variation. Future work aims at an experimental implementation of the proposed algorithm and at experimentally proving its usefulness in improving the perception of the remote environment. We will also focus on the use of the proposed control strategy for Internet based communication channels where the communication delay is variable and some packets can get lost.

\section{REFERENCES}

[1] S. Stramigioli, A. van der Schaft, B. Maschke, and C. Melchiorri, "Geometric scattering in robotic telemanipulation," IEEE Transactions on Robotics and Automation, vol. 18, no. 4, 2002

[2] A. van der Schaft, $L_{2}$-Gain and Passivity Techniques in Nonlinear Control, ser. Communication and Control Engineering. Springer Verlag, 2000.

[3] S. Stramigioli, C. Secchi, A. van der Schaft, and C. Fantuzzi, "Sampled data systems passivity and discrete port-hamiltonian systems," IEEE Transactions on Robotics, vol. 21, no. 4, pp. 574-587, 2005.

[4] J.-J. Niemeyer, G. ans Slotine, "Telemanipulation with Time Delays," International Journal of Robotics Research, vol. 23, no. 9, pp. 873-890, September 2004.

[5] N. Chopra, M. Spong, S. Hirche, and M. Buss, "Bilateral teleoperation over the internet: the time varying delay problem," in Proceedings of American Control Conference, vol. 1, Denver, Colorado, USA, June 2003.

[6] D. Lee and M. Spong, "Passive bilateral control of teleoperators under constant time-delay," in Proceedings of IFAC world Congress, Prague, Czech Republic, July 2005.

[7] P. Arcara and C. Melchiorri, "Position drift compensation for a passivitybased telemanipulation control scheme," in Proceedings to Mechatronics Conference, Enschede, The Netherlands, June 2002.

[8] S. Stramigioli, Modeling and IPC Control of Interactive Mechanical Systems: a coordinate free approach, ser. LNCIS. Springer, London, 2001.

[9] C. Secchi, "Interactive robotic interfaces: a port-hamiltonian approach," Ph.D. dissertation, University of Modena and Reggio Emilia, 2004, available at http://www.dismi.unimore.it/download/thesis.pdf.

[10] G. Niemeyer and J. Slotine, "Stable adaptive teleoperation," IEEE Journal of Oceanic Engineering, vol. 16, no. 1, pp. 152-162, 1991.

[11] J.-J. Slotine and W. Li, Applied Nonlinear Control. Prentice Hall, 1991. 